\section{Salud sexual y factores de riesgo: aportes etnográficos de jóvenes del Caribe costarricense}

RESUMEN

El artículo resume notas de campo y entrevistas a profundidad realizadas a jóvenes de la comunidad turística de Puerto Viejo, en el Caribe Sur costarricense, sobre el tema de salud sexual y riesgo. Se argumenta que sus visiones acerca del cuerpo, de la intimidad y de la persona extranjera, además de ser fundamentales al distinguir las previsiones que deben tener en sus relaciones sexuales, se encuentran enraizadas en distinciones de clase, de género y de raza. A partir de sus valoraciones en cuanto al uso del condón masculino, se ejemplifica cómo el empleo de técnicas de investigación cualitativas puede ofrecer insumos al debate tradicional sobre factores de riesgo desarrollado en el campo de la salud pública.

Palabras Clave

Riesgo; salud sexual y reproductiva; adolescencia y juventud; Caribe sur Costa Rica

\section{Sexual Health and Risk Factors: Ethnographic Contributions of Young People from Costa Rica's Caribbean South}

ABSTRACT

The article summarizes data from field notes and in-depth interviews on the subject of sexual health and risk with young people in the tourist community of Puerto Viejo -located in Costa Rica's South Caribbean. It is argued that their views about the body, intimacy, and foreigners, are not only key to distinguishing the care they should have regarding their sexual relations, but also constitute reasons differentiated by class, gender and race. Besides, taking as example their assessment about the use of male condos, it is shown how the use of qualitative-based analysis can provide input to the traditional debate developed in the field of public health about risk factors.

KEY WORDS

Risk; reproductive and sexual health; Adolescence and youth; Costa Rica's Caribbean South
Artículo recibido:

26 de julio del 2015.

Evaluado:

18 de agosto del 2015.

Aceptado:

14 de noviembre del 2015.

Costarricense. Obtuvo su Doctorado en Sociología por la Universidad de Windsor, Canadá. Se desempeña como profesor de la Escuela de Sociología de la Universidad de Costa Rica, donde imparte cursos sobre sociología de la salud y análisis de políticas públicas. Además, es investigador del Instituto de Investigaciones Sociales (IIS) en esta misma universidad. Correo electrónico: mauricio.lopez@ucr.ac.cr

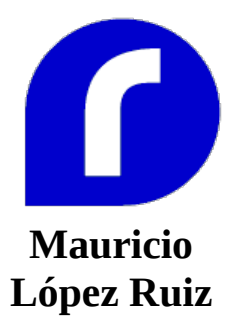




\section{Salud sexual y factores de riesgo: aportes etnográficos de jóvenes del Caribe sur costarricense}

1. Siguiendo los estándares de la Organización Panamericana de la Salud (OPS), se define a la población joven como aquella que va de los $10 \mathrm{a}$ los 24 años. Dentro de este grupo, se encontrarían los preadolescentes (de $10 \mathrm{a}$ 14) y los adolescentes (de15 a 24).
El desarrollo de estrategias de atención a las poblaciones adolescentes y jóvenes constituye uno de los principales retos en los cuales deben trabajar nuestras sociedades contemporáneas. ${ }^{1}$ Esto debe llamar nuestra atención. Estamos en presencia de un grupo etario que representa más del cincuenta por ciento de la población mundial, el cual, a pesar de ser percibido como saludable, se muestra altamente vulnerable a problemas de salud determinados por el entorno cultural y social, a saber: consumo de drogas, patrones de nutrición inadecuados, trastornos alimentarios, homicidios y suicidios, así como el contagio de infecciones de transmisión sexual (ITS), incluyendo el virus de la inmunodeficiencia adquirida $(\mathrm{VIH})$ o complicaciones derivadas del embarazo, parto y puerperio (UNFPA, 2013, p.10; CCSS, 2014).

En Latinoamérica, el embarazo durante la adolescencia y el contagio de ITS han sido preocupaciones centrales en las agendas de política formuladas en torno a la salud sexual. Se estima que, al finalizar su adolescencia, aproximadamente la mitad de hombres y de mujeres habrán sostenido este tipo de relaciones (UNFPA, 2014); por lo tanto, constituye uno de los acontecimientos más relevantes que será experimentado durante esta etapa de la vida.

Así pues, las autoridades de salud han tratado de impulsar, en mayor o en menor medida, acciones que le permitan a este grupo etario desarrollar una vida sexual segura. Como parte de esta tarea, la identificación de factores de 
riesgo referidas al inicio de relaciones sexuales (entendidas en términos de penetración anal, vaginal, así como sexo oral) ha ocupado un lugar prioritario.

Ahora bien, el establecimiento de parámetros sobre riesgo en el ámbito de la salud sexual puede ser un ejercicio un tanto engañoso. En realidad, siempre resulta complicado brindar definiciones universales u homogéneas sobre dichos tópicos; sin embargo, estas existen. Nacemos en una cultura donde se nos ha enseñado no solo a reproducir implícita o explícitamente algunas de ellas, sino también a tomarlas como base de nuestras prácticas comunes y corrientes. Y esto, que define nuestro carácter social, también nos condiciona a tomar ciertas definiciones como naturales, como algo que en lugar de obedecer a una construcción colectiva se instala más bien en un orden preestablecido de las cosas.

Este artículo tiene por objeto, justamente, contrastar ideas sobre el riesgo, desarrolladas por jóvenes de la comunidad turística de Puerto Viejo de Talamanca (en el distrito de Cahuita, localizado en la provincia de Limón), con aquellas utilizadas de manera oficial en el Área de Salud de Talamanca. ${ }^{2}$

En tanto caso de estudio, Puerto Viejo reúne algunos rasgos distintivos a los cuales se les debe prestar cuidado. Primero, estamos en presencia de una antigua comunidad rural cacaotera, embarcada desde la pasada década de los años ochenta en una reconfiguración económica que la ha llevado a transformarse en una de las principales atracciones turísticas de Costa Rica, pues convoca principalmente a turistas de origen norteamericano y europeo quienes buscan vivir, conocer y experimentar "el estilo de vida caribeño" (Frohlick, 2012).

A pesar de su atractivo turístico y de sus riquezas naturales, toda la parte costera del cantón de Talamanca muestra un importante rezago socioeconómico, que se expresa en carencias relativas a infraestructura, espacios públicos y servicios básicos; en la escasez de fuentes de empleo formal bien remunerado; así como en bajos niveles de escolaridad en secundaria y en chances de movilidad social (Carmiol, Chacón, Vargas y Walker, 2014; Molina, 2007). El turismo, en estos últimos años, ha representado para las nuevas generaciones una opción mediante la cual obtienen ingresos económicos, acceden a bienes de consumo y mejoran su estatus societal.

Segundo, y esto fue relevante para los efectos de la presente investigación, el contacto frecuente que tienen sus lugareños con distintos grupos sociales, en virtud de esta nueva economía de servicios, también ha influido en la vida íntima de sus pobladores. En este mismo orden de ideas, el abuso de drogas y los encuentros sexuales casuales incrementan la exposición a sus pobladores al contagio de ITS. En este sentido, resulta común encontrar jóvenes involucrados en relaciones íntimas con turistas que, según explica Frohlick
2. El sistema público de salud costarricense se divide geográficamente en áreas rectoras de salud (a cargo del Ministerio de Salud) y áreas de salud (a cargo de la Caja Costarricense de Seguro Social-CCSS). En este artículo, se hablará únicamente de área de salud (englobando así las áreas administrativas y de prestación de servicios). 
3. Este estudio se desprende de un trabajo colaborativo realizado con la Dra. Susan Frohlick, de la Universidad de Manitoba, Canadá. Su realización fue posible gracias a fondos otorgados por el programa LACREG (Canada-Latin America and the Caribbean Research Exchange Grants); el valioso trabajo de asistencia de la socióloga Carolina Meneses; y el apoyo del Instituto de Investigaciones Sociales de la Universidad de Costa Rica.

4. Asimismo, y para efectos de brindar información básica sobre el desarrollo de políticas en salud sexual en el área de salud de

Talamanca, hemos incluido un par de menciones producto de las entrevistas al personal de la CCSS y al Ministerio de Salud. Actualmente, se prepara otro artículo en el cual se profundiza lo anterior.
(2012, 2013), están mediadas por transacciones de bienes materiales y simbólicos.

De este modo, sus sexualidades se encuentran, de una u otra manera, influidas por sus encuentros con turistas domésticos e internacionales, así como por migrantes nacionales e internacionales allí afincados. Si se suman grupos indígenas de la zona de Talamanca y la misma población afrodescendiente del lugar, tenemos como resultado un espacio de interrelación multicultural que no es fácil de encontrar en otras regiones del país.

Por último, y esto es un indicador indirecto acerca de iniciación sexual, en esta zona se presenta una destacable cantidad de embarazos en menores de 15 años, así como un promedio de madres menores de edad que, prácticamente, dobla el promedio registrado en el país (CCSS, 2013, p. 33; INEC, 2011).

Los hallazgos de la investigación, expuestos a continuación, forman parte de un estudio piloto cuyo propósito general fue analizar el acceso a servicios en materia de salud sexual por parte de residentes de dicha comunidad. ${ }^{3}$ Para este artículo, se presenta información recolectada en torno al tema de riesgo.

La principal fuente de datos utilizada proviene de notas de campo y de entrevistas a profundidad conducidas a finales del año 2012 y durante breves periodos de estancia (usualmente de dos semanas) que sucedieron a lo largo del 2013. En total, 19 entrevistas fueron hechas a jóvenes provenientes de Puerto Viejo, de entre 18 y 25 años (13 mujeres y 6 hombres). En su conjunto, dichas entrevistas expresan vivencias de quienes han crecido en la zona, y se han desenvuelto en el mercado de actividades de servicio (por ejemplo: meseras, dependientes de tiendas, cocineras, guías turísticos o artesanos), de ingresos económicos bajos, que no pudieron terminar sus estudios formales en secundaria; y en el caso de varias mujeres, jóvenes madres de familia. ${ }^{4}$

La muestra utilizada no fue seleccionada de manera aleatoria, sino utilizando el procedimiento de "bola de nieve". En un primer momento del estudio, se utilizaron contactos personales para ubicar a posibles candidatos, quienes fueron accediendo. Recomendaron, a su vez, a otras personas para entrevistar. Esta labor de reclutamiento de colaboradoras y de colaboradores fue facilitada por nuestra asistente de investigación, quien ha vivido en dicha comunidad durante los últimos ocho años. Las entrevistas recolectadas se transcribieron y se analizaron mediante el software NVivo 10, lo cual ayudó en el trabajo de codificación, de comparación y de sistematización de notas de campo y narraciones.

La exposición de contenidos está dividida en cinco secciones centrales. En la primera, se hacen anotaciones preliminares acerca del tema de riesgo, que permitirán posteriormente examinar la noción de factores de riesgo que se 
desarrolla en el Área de Salud de Talamanca. Luego, se explica de modo breve cómo dicha noción se encuentra en las acciones de política en salud sexual allí adoptadas. Esto sirve de preámbulo para abordar ideas sobre riesgo y salud sexual compartidas por jóvenes de Puerto Viejo, tema de las restantes secciones. Si bien en términos estadísticos, la muestra no tiene un carácter representativo para la región Caribe sur, es posible afirmar que mucha de la información obtenida permitirá sentar las bases para estudios que profundicen en la temática, así como en la formulación de algunas recomendaciones de política social.

\section{Sobre la idea de riesgo: aproximaciones conceptuales}

El debate en torno al riesgo ha ocupado un lugar preponderante en el campo de la salud pública. Dada la gran influencia que ha tenido la epidemiología tradicional en este campo, sobre todo en el desarrollo de estudios observacionales que dan cuenta de patrones colectivos de morbilidad y mortalidad, el riesgo se ha tematizado en términos de factores de riesgo. Los factores de riesgo se han entendido como "una conducta personal, una exposición al medioambiente, o una característica innata que, con base en la evidencia científica, se reconoce como asociada a algún, o algunos, padecimientos en salud" (Porta, 2013, p. 218). Este punto de vista, valga la acotación, parte de distinciones biomédicas en las cuales la salud se entiende como la ausencia de una enfermedad resultante de alguna anomalía de tipo genético, químico o fisiológico, que daña ya sea las funciones o la estructura del organismo humano (Martínez, 2011, p. 50).

Respecto del último aspecto, dichos factores constituirían las causas directas o indirectas que activan dichas anomalías y aumentarían la probabilidad de que un individuo se enferme o muera dentro de un lapso determinado o a cierta edad (Almeida, Castiel y Ayres, 2009; Porta, 2013).

En este sentido, el uso y el contacto cotidiano con elementos tóxicos, como el plomo; las vulnerabilidades congénitas, heredadas de generación en generación; o bien la elección de estilos de vida caracterizados por el consumo de tabaco o la ausencia de actividad física regular se encuentran entre algunos de los principales factores de riesgo en torno a los cuales distintos sistemas de salud han movilizado una enorme cantidad de recursos.

De hecho, como ha sido señalado por Alaszewski y Brown (2012), Brown y Heyman (2012), Lupton (2012), Porter (1999) y Smallman (2007), entre otras personas, los factores de riesgo han permitido crear consensos normativos y valorativos capaces de justificar la formulación e implementación de ciertas políticas en salud. Difícilmente, esos consensos hubieran sido establecidos apelando únicamente a otro tipo de razones o principios, como el bienestar, la equidad o el reconocimiento de derechos. 
En el campo de la salud pública, esta noción de factores de riesgo se circunscribe a una perspectiva que, siguiendo a Drew (2012) y a Lupton (2013), podría denominarse como técnico-científica. En su formulación, existen aportes de varias disciplinas como la epidemiología, la estadística, el campo actuarial o la economía, las cuales no solo comparten como meta la construcción de modelos predictivos para calcular la magnitud y la severidad que tendría en una población la ocurrencia de un evento adverso, sino también la ambición de diseñar las estrategias preventivas o paliativas que deben seguir las personas futuras afectados.

Dentro de esas disciplinas, además, es poca la atención prestada a la forma en que histórica y culturalmente diversos eventos han ido adquiriendo (o dejado de adquirir) un estatus de riesgo. En consecuencia, y a pesar de sus contribuciones al campo de la salud pública, sus respectivas nociones de riesgo tienden a ser naturalizadas y universalizadas.

Las ciencias cognoscitivas, especialmente la psicología, también han contribuido a la noción de factores de riesgo trabajada en el campo de la salud pública. La construcción de modelos predictivos también constituye una de sus metas, aunque en este caso, con miras a estudiar respuestas cognitivas y conductuales de las personas ante riesgos específicos.

Al estar adscritos a una perspectiva técnico-científica, estos modelos son presentados por parte de la comunidad investigadora como hechos naturalizados, que dan cuenta de las actitudes y de percepciones que conducen a las personas a asumir riesgos (según determinadas pruebas psicométricas). Al final de cuentas, lo que prima en este trabajo es saber qué tan acertadas 0 equívocas resultan las respuestas de los individuos en función de ciertos riesgos sometidos a prueba, para de esta manera, planear intervenciones capaces de educar correctamente a individuos y a grupos sociales (Lupton, 2013, p.29).

Existen otras perspectivas alternativas acerca del riesgo. Para quienes formamos parte del gremio de la sociología, los nombres principales que inmediatamente vienen a la mente serían los de Ulrich Beck y Anthony Giddens, sociólogos cuya obra se popularizó durante la década de 1990. A pesar de sus diferentes abordajes, ambos autores parten de la tesis de que las sociedades industriales (entiéndase las de mayores ingresos en Europa y en Norteamérica) se encuentran inmersas en una fase histórica que las estaría redefiniendo en términos de sociedades de riesgo. Una característica elemental de estos conjuntos sociales sería el establecimiento de estrategias dirigidas a gestionar daños derivados de sus propios procesos de modernización, algunos de los cuales a menudo resultan irreversibles y de impacto general (por ejemplo, el calentamiento global).

Conforme van aumentando los riesgos y la conciencia que sobre ellos tienen personas y los grupos sociales, aumenta también la desazón que surge en 
los individuos al vivir en mundo signado por la incertidumbre. Se inaugura así una fase reflexiva de crítica y de autoconfrontación sobre los efectos colaterales que está trayendo la modernidad, en donde el conocimiento y el discurso experto estarían jugando un papel central (Beck, 1998, 2009, 2011; Giddens, 2009; Giddens y Sutton, 2014). ${ }^{5}$

5. En este debate, se diferencia conceptualmente el riesgo del peligro. El

peligro es un daño del cual debe cuidarse y que se atribuye a causas fuera del control de quienes estén implicados, mientras que el riesgo supone siempre un daño producido por decisiones colectivas y personales.

riesgo es algo que depende de conocimientos y de discursos precedentes, sean estos catalogados como saberes expertos o saberes acuñados por la gente común y corriente (algo que distancia a esta autora de la idea de reflexividad mencionada anteriormente). Crítica acérrima de enfoques basados en la figura ideal del actor racional, Douglas (1994; 1996).

Douglas $(1994 ; 1996)$ afirma que toda concepción sobre el riesgo es compartida culturalmente (obedeciendo así a sistemas compartidos de saberes); y no, el producto único de conocimientos y percepciones individuales. Al mismo tiempo, las respuestas culturales al riesgo sirven para establecer reglas colectivas de comportamiento y para trazar límites simbólicos que distinguen a los miembros de una comunidad.

El reproducir prácticas riesgosas, lo ubica a uno en categorías sociales no confiables; supone una transgresión que afecta al resto de la comunidad y señala, a la vez, fallos morales sobre los cuales se debe impartir justicia. Sobre quienes haya recaído la etiqueta de riesgo (estar en riesgo o ser un riesgo para los demás), se posan generalmente, también estatus devaluados, ligados a la debilidad, a la pasividad o a la vulnerabilidad, de los cuales debe resguardarse el ordenamiento social.

A continuación, se retomarán algunos de estos elementos conceptuales, con el fin de analizar cómo la idea de riesgo ha sido construida por funcionarias y funcionarios del Área de Salud de Talamanca. El examen de su conocimiento, respecto del tipo de daños que se ciernen sobre la juventud en salud sexual y el ejercicio de reflexividad que le acompaña, resulta un buen indicador de la forma de gestionar riesgos por el cual se han decantado en épocas recientes.

\section{Salud pública y el primado de la noción de factores de riesgo}

La formulación e implementación, a mediano y a largo plazo, de políticas dirigidas a la salud sexual de la población joven constituyen hoy en día una de 
las grandes tareas pendientes del sistema público de salud costarricense. En el caso específico del Área de Salud de Talamanca, durante el transcurso del último decenio, esta situación ha generado un puñado de iniciativas aisladas sobre esta materia; algunas de más largo aliento que otras, con un limitado impacto en este grupo etario y una orientación más curativa que preventiva (López-Ruiz, 2014).

Sin entrar a detallarlas, las labores realizadas en dicha área de salud sobre esta materia heredan de la epidemiología tradicional un marcado énfasis biomédico. Por ello, y a pesar de sus respectivas diferencias, tienden a ubicar en sus agendas dos preocupaciones predominantes, a saber: el embarazo adolescente y el contagio de ITS y de VIH.

En esta línea de trabajo, el debate sobre el tema de riesgo se ha ido construyendo en función de dos tipos de comportamientos individuales: en primera instancia, las relaciones sexuales (entendidas, como dijimos en la introducción, en términos de penetración anal y vaginal, así como de sexo oral); y segundo, el uso compartido de jeringas o de agujas en consumidores de drogas intravenosas (MINSA, 2012; CCSS, 2013a).

Ambos comportamientos, sobre todo el primero (en virtud de su conexión con el embarazo durante la adolescencia), han sido tratados por funcionarios de salud (CCSS, 2013b) en cinco tipos de factores de riesgo:

- La práctica de relaciones sexuales sin conocer cómo sucede y cómo se puede impedir, biológicamente, un embarazo o el contagio de ITS y VIH;

- La ausencia del uso de métodos de barrera, a saber: el condón masculino al tener relaciones, especialmente en la penetración pene-ano (dada la sensibilidad a fisuras que presenta esta parte del cuerpo);

- El descenso en la edad de inicio de relaciones;

- El aumento en el número de parejas íntimas en el transcurso de la vida, sean estas en el contexto de monogamia serial, o bien, de relaciones concurrentes; $y$

- El aumento de encuentros sexuales casuales.

En cuanto a estas conductas de riesgo, vale la pena mencionar que su identificación no se sustenta en estudios sistemáticos sobre las sexualidades de la persona joven, sino más bien en el conocimiento e impresiones personales que han venido acuñando quienes trabajan en el sistema público de salud del Caribe sur (López-Ruiz, 2014). Aquí debe tomarse en consideración que este tipo de factores de riesgo son citados constantemente en la literatura sobre salud sexual, por ende, forman parte habitual de los tópicos que acaparan la atención de diversos sistemas de salud alrededor del globo, así como de los 
principales organismos internacionales adscritos al campo de la salud pública (Ingham y Aggleton, 2006; OMS, 2009; y Aggleton y Parker, 2012).

A pesar de la relevancia que tiene este debate, la noción de factores de riesgo no suele acompañarse por explicaciones capaces de situar comportamientos de riesgo en sus respectivos entornos sociales y culturales. En términos analíticos, esto tiene una consecuencia importante para los efectos del presente artículo. Desde el punto de vista de las autoridades de salud pública, el comportamiento de la población joven muestra un carácter disfuncional que es el producto "natural" de una mezcla entre actitudes inmaduras o indolentes, por una parte, y del declive de arreglos colectivos asociados al cortejo y al noviazgo, a la fidelidad y a la familia tradicional de tipo nuclear. Más importante aún, estas "malas" elecciones serían de orden irracional, las cuales se ubicarían en un dominio de responsabilidad moral individual (López-Ruiz, 2014).

Siguiendo esta lógica racional, la gestión de riesgos en salud sexual desarrollada en el Área de Salud de Talamanca se ha orientado a modificar las conductas de riesgo previamente identificadas. La persona joven aparece dentro sus propuestas preventivas en términos de actores a quienes se debe educar acerca del sinsentido de sus acciones; para así, hacerles entrar en razón sobre sus errores (entrevista: CCSS). Será entonces una de las tareas encomendadas a funcionarios de salud pública la entrega de información adecuada que les permitan calcular los costos personales de asumir ciertos riegos. Con ello, se establece una relación proporcionalmente inversa entre acceso a conocimiento experto y el cuidado que, en buena teoría, deberían ejercer sobre sí mismos los individuos, estrategia que en la literatura anglosajona se conoce bajo el nombre de Health Belief Models (Adam,2006; Bloor, 1995 y Schneider, 2011).

Las siguientes secciones del presente artículo están dedicadas a contrastar esta postura oficial sobre el riesgo con ideas expresadas por jóvenes residentes en Puerto Viejo. Esta población joven menciona tanto sus propias vivencias como las de sus grupos de pares. Asimismo, nos ayudarán a argumentar que el riesgo no debe ser entendido exclusivamente como un evento "objetivo", sometido a cálculo racional por parte de los individuos. Por el contrario, debe ser construido y negociado en términos relacionales, es decir, en función de cómo acostumbran las personas a interrelacionarse y de la formación de significados y de emociones que derivan de dichas experiencias.

\section{Conversando sobre factores de riesgo ${ }^{6}$}

6. Tanto en esta sección como en las dos que siguen, las citas provienen de las entrevistas realizadas a jóvenes de la comunidad de ¿Qué tanto ha calado la noción de factores de riesgo expuesta anteriormente en jóvenes residentes de Puerto Viejo? El presente trabajo permitió elaborar 
7. En cuanto al inicio de relaciones sexuales, las personas entrevistadas mencionaron rangos de edad que irían de los 10 a los 14 años, por debajo del promedio nacional ubicado en 15 años, según la última Encuesta Nacional de Salud Sexual y Reproductiva (ENSSR) realizada en Costa Rica en el 2010. Evidentemente, las apreciaciones de quienes entrevistamos deben someterse a un análisis detallado, para ser debidamente verificadas. una primera respuesta, a pesar de lo novedoso que resultaba para nuestra población joven de colaboradores discutir acerca de su salud sexual.

Como se ha mencionado, las únicas oportunidades en las cuales esas personas habían escuchado a otras hablar públicamente sobre este tema habían sido en el contexto de ferias de la salud desarrolladas por la CCSS, o bien en el ámbito escolar, cuando alguno de sus profesores de biología en el colegio les explicaban el "funcionamiento del sistema reproductivo masculino y femenino" y los órganos que lo integran.

Esta información era, por lo general, recordada de manera vaga, aunque algunas nociones elementales sobre la existencia de los condones masculinos, así como métodos de planificación (sobre todo, pastillas anticonceptivas) se conservan en su memoria. Particularmente, cuando iban acompañadas de consejos, como: cuidarse de tener que "criar un bebé tan joven" en el caso de las mujeres, o bien de "que le peguen a uno alguna enfermedad" en el caso de los hombres.

Ahora bien, es necesario tener en cuenta que no todas las personas entrevistadas tuvieron la fortuna de cursar estudios secundarios o de tener contacto con alguna feria de la salud; por lo tanto, su experiencia de aprendizaje sobre este tema se dio de otra manera.

Según los datos analizados, la obtención de una noción similar a la oficial (es decir, apegada a la noción de factor de riesgo) tuvo su principal anclaje en el espacio del consultorio médico, sobre todo en el caso de las mujeres que habían quedado embarazadas. La interacción con personal médico les abrió la puerta para adquirir alguna instrucción formal acerca del uso de métodos anticonceptivos como medio para prevenir embarazos; para darse cuenta de la importancia que tienen los chequeos regulares en ginecología; y de paso, para aprender que el uso de condones resulta un medio altamente efectivo para protegerse contra enfermedades transmitidas al tener relaciones sexuales.

Esta adquisición de información sobre el tema de embarazo e ITS ejemplifica bien la orientación curativa que aún persiste en las acciones coordinadas desde el sistema público de salud. Como nos dijo una de nuestras entrevistadas, si bien es cierto la gente empieza a tener una "vida sexual activa" a edades tempranas, no es sino hasta que pasan este tipo de cosas "que reaccionan y empiezan a cuidarse."7 Para las mujeres que no habían estado embarazadas y para quien no hubiera estado en contacto con el espacio médico por motivo de una ITS (nadie mencionó haber contraído alguna vez una de ellas), la idea de lo que podía constituir un riesgo en materia de salud sexual había sido aprendido "en la calle," tal y como uno "aprende todo en la vida."

Para estas personas, las fuentes de información más comunes fueron, según su orden de importancia: a) opiniones y vivencias personales de amigos, her- 
manos mayores o primos que habían contraído enfermedades "raras" por no usar condón, en el caso de los hombres; b) historias sobre complicaciones en embarazos tenidos a edades tempranas, compartidas entre amigas, tías y hermanas, en el caso de las mujeres; c) menciones referidas al peligro de contraer gonorrea, sífilis, o SIDA escuchadas en ciertos programas y noticiarios radiales y televisivos. Al menos para las personas entrevistadas, el mundo del internet y sus redes sociales no constituye todavía un lugar donde informarse acerca de salud sexual.

Volviendo a la pregunta inicial de este apartado, tendríamos que la noción oficial sobre riesgo planteada desde el sistema público de salud no ha encontrado aún eco en la mayoría de jóvenes. A lo anterior, contribuyen tanto su exclusión del sistema educativo como su ausencia a consulta médica relativa a embarazo o a contagio evidente de alguna ITS. Este último punto, nos llevará a otro par de temas conexos.

Como hemos sugerido, una noción "oficial" sobre factores de riesgo constituye uno entre otro tipo posible de saberes. Para abordar este conocimiento alternativo, conversamos con nuestras y nuestros entrevistados acerca de tres tópicos concretos: qué consideraban normal en su vida sexual; con quién creían inconveniente tener sexo; y en especial, qué tipo de cuidados debían tenerse. Sus primeras impresiones y consejos, permiten ilustrar un tema vital en el marco de las preocupaciones centrales del sistema público de salud, a saber, el uso del condón masculino.

\section{Otras posturas frente al riesgo: el primado del cuerpo y la intimidad}

Más que racionales, las personas tendemos a ser razonables, es decir, gran parte de nuestras acciones ( $y$ el sentido que les damos) escapan a la mera búsqueda del bienestar y del interés propio, o bien al cálculo sopesado de los costos y de los beneficios que nos podrían traer. En consecuencia, al platicar con nuestras y nuestros entrevistados sobre los cuidados que uno debería seguir al tener relaciones sexuales, salieron a la luz las siguientes razones que suelen pasarse por alto en el debate sobre factores de riesgo.

El primer tipo de razonamiento se centró en la dimensión corporal. Esto no resulta sorprendente si se toma en cuenta que la idea de salud del grupo entrevistado (como la de la mayoría de las personas) era negativa, es decir, centrada en la enfermedad; condición que en una cultura como la nuestra supone, implícita o explícitamente, un declive en la autonomía e integridad orgánica. En este sentido los y las entrevistadas aconsejaron lo siguiente: a) fijarse si la persona era limpia; b) si se veía sana; c) si tenía algún tipo de mal olor corporal; y por último, c) si tenía alguna anormalidad en sus genitales. 
Desde el punto de vista anterior, el riesgo se conceptualiza en el cuerpo. Aquellos cuerpos que no muestran una capacidad de regular su autonomía e integridad serían puestos en entredicho. Por consiguiente, si se encontraban esos signos de impureza, se nos sugería que justificaba las dudas o temores sobre la pareja sexual. En situaciones como esta, lo mejor sería abstenerse, o bien utilizar (o sugerir) un condón. Igualmente, y aunque se haya usado preservativo en estas circunstancias, la mayoría de jóvenes consideró adecuado tener algunos cuidados extra en relación con el propio aseo corporal después de tener sexo. Bañarse y lavarse bien con agua y jabón los genitales o el ano fueron los hábitos de limpieza más citados.

Siguiendo a la antropóloga Deborah Lupton, dichas costumbres de aseo corporal pueden interpretarse como rituales de purificación que nos permiten lidiar con potenciales ansiedades y disgustos producidos por el contacto con partes del cuerpo "codificadas culturalmente como sucias y contaminantes"; y por lo tanto, dañinas (Lupton, 2013, p.180). Un razonamiento similar a este fue compartido por uno de nuestros entrevistados, quien recomendaba el jugo de limón como medio de limpieza y como forma de identificar si la otra persona padecía alguna enfermedad: "Usted parte un limón en dos. Usted se lo frota a ella en su cosa, y/o ella me lo frota a mí. Si arde y rápidamente se pone rojo, entonces usted tiene una enfermedad." En caso de que la pareja no quisiera realizar este ejercicio, eso también sería un indicador de que no era de fiar, porque "sabía lo que le espera". La apreciación sobre la pureza del cuerpo del otro, así como la limpieza del propio cuerpo resultaron centrales para comprender qué tan segura se siente una persona al tener relaciones sexuales.

Un segundo tipo de razonamiento tenía que ver más con el ámbito de la intimidad y su papel en las interacciones sexuales, entendiéndolo como un vínculo afectivo cercano en términos físicos y emocionales, tal y como lo definen Jamieson (2011) y Zelizer (2007). En este aspecto, la iniciación sexual del grupo entrevistado fue posible con personas que tenían tiempo de conocer (les ligaba algún grado de buena amistad o enamoramiento) y con quienes ya tenían una vida sexual activa.

En el caso de las mujeres, el hecho de tener una conexión afectiva con una persona experimentada resultó necesaria para sentirse "seguras," para calmar la mezcla de sensaciones de incertidumbre, ansiedad, excitación, incomodidad y placer, que pueden ligarse a prácticas como el besarse, el magreo o el coito. En el caso de los hombres, esta conexión afectiva en el ámbito de la intimidad resultaba de ayuda en cuanto al control de emociones relativas al temor que sentían de "fallarle" a su pareja sexual, de mostrarse "débiles," de no ser capaces de retardar su eyaculación, de no disfrutar lo que se hacía, y por ende, de no estar aprendiendo a ser buenos amantes.

Sensaciones similares a las anteriores, en las cuales el lazo entre intimidad y seguridad se encontraba mediado por distinciones de género, fueron también 
reportadas como parte integral de interacciones sexuales posteriores y como un factor para valorar si el uso de preservativos era o no necesario.

Entre las personas que habían utilizado el condón, se marcaron dos posiciones. Por un lado, los hombres admitieron que no les gustaba mucho ponérselos, a pesar de tener cierta conciencia sobre la protección que ofrecían en relación a "enfermedades raras". Su uso resultaba un doble estorbo: algo que no solo limitaba la obtención de placer, sino también su buen desempeño en la cama; por lo tanto, se convertía en un "factor de riesgo" para su virilidad. Se nos dijo que su uso era innecesario cuando uno "llevaba tiempo de conocer a sus parejas sexuales", ya fuera "personalmente o en la cama." En estos casos, solo se pondrían un condón siempre y cuando se les impusiera como un requisito para tener sexo. Asimismo, el que "a uno le pidan lo anterior", también podría significar que la pareja no le estaba teniendo la confianza que "se merece". 8

Por otra parte, nuestras entrevistadas expresaron que no todas sus parejas habían usado condón. En sus narraciones, puede notarse cómo su utilidad se encontraba más ligada al tema de evitar embarazos que a protegerse contra alguna enfermedad. Lo anterior tuvo dos implicaciones básicas. Primero, que los condones no fueran vistos como necesarios en el sexo anal u oral. En caso de ser requeridos en el coito vaginal, se esperaba que fueran los hombres quienes los compraran y los trajeran consigo al tener sexo (algo que, de acuerdo a la nota al pie número ocho, no necesariamente querrían o podrían hacer todos los hombres) ${ }^{9}$.

Segundo, que la importancia de usarlos disminuyera en función del tiempo de conocer a sus potenciales parejas sexuales. Sin embargo, a diferencia de sus contrapartes masculinas, esto sucedía porque no les importaba jugarse algún chance de quedar embarazadas, siempre y cuando existiera algún tipo significativo de conexión íntima con la pareja sexual. Dicho enlace partía de vínculos afectivos, por ejemplo: tener una "buena amistad," estar "enamorada," haber recibido apoyo o el poder construir una relación de mutua convivencia.

Un aspecto por resaltar es la reacción de las mujeres ante el comportamiento infiel de los hombres locales. Si bien ellas aceptaban abiertamente que a ellos les "daba igual tener 2, 3, 4, 5 mujeres", esto no se tradujo todas las veces en una necesidad de adoptar mayores precauciones en su vida sexual ni de preguntarles si utilizaban condón con sus otras parejas.

Respecto de este tema hay mucha tela que cortar, sobre todo, cuando en el fondo lo que se encuentra en juego son relaciones de dominación masculina en las cuales se mezclan desde condiciones de desventaja socioeconómica (mujeres que dependen de los ingresos de sus parejas, como lo eran algunas de nuestras entrevistadas) hasta diferencias culturales de orden patriarcal que hacen pasar por natural este tipo de prácticas.
8. También, es necesario tener en cuenta que algunos hombres mencionaron que sus ingresos económicos no les alcanzaban para costearlos. Teniendo tantas necesidades, se nos dijo, su compra no representaba una prioridad en sus vidas.

9. Acá debe tomarse nota que, para algunas entrevistadas, ir al súper o a la farmacia a comprar un condón sería algo mal visto "en el pueblo", dada la existencia de gente "prejuiciosa". Por ello, "no se animan" a comprar pastillas ni condones "porque no quieren imaginarse después lo qué dirá la gente". 
En todo caso, el que los hombres tuvieran parejas sexuales simultáneas (sin conocer si en sus relaciones mediaba o no el uso de preservativos) era algo tolerado, siempre y cuando los hombres no tuvieran hijas o hijos con ellas. El cruce de este límite supondría un "irrespeto" a ellas como parejas, a la confianza y a la seguridad depositada en esa relación íntima, así como un claro indicador de que el comportamiento sexual de los hombres "no conduce a nada bueno".

\section{El riesgo que supone el encuentro con turistas}

Las apreciaciones y valoraciones sobre el cuerpo y la intimidad, como las expuestas anteriormente, constituyen solo parte de los recursos con los que cuentan las personas para decidir si los preservativos resultan necesarios al involucrarse sexualmente con alguien. Esas valoraciones le dan a la gente razones que les ayudarán a conducir su vida sexual en determinados contextos de interacción social. Asimismo, atributos ligados al estatus social, como la pertenencia a cierta clase económica o distinciones raciales, también cumplen un papel relevante al categorizar a las personas en distintos grupos de riesgo. En el caso de la presente investigación, como se desarrollará en seguida, este tema salió a la luz al hablar sobre el impacto que tiene el turismo y el encuentro constante con personas extranjeras, en las prácticas sexuales desarrolladas en su comunidad.

En opinión del grupo entrevistado existe la noción generalizada de que la vida nocturna de Puerto Viejo, al compararla con la de otras playas y comunidades circundantes, ofrece un menú de diversión y esparcimiento que incluye, entre otros, los encuentros sexuales casuales con turistas. Su llegada, sobre todo en la época de temporada alta, conlleva un aumento en las probabilidades que tienen las y los lugareños de tener sexo.

Como parte de lo recién descrito, una práctica común en la zona ha sido el establecimiento de relaciones sexuales de corto o mediano plazo entre hombres afrodescendientes jóvenes y extranjeras (europeas y norteamericanas) de mediana edad. Si bien hace falta estudiar con más detalle qué tan extendida resulta esta práctica, el trabajo que ha venido realizando la antropóloga canadiense Susan Frohlick $(2012,2013)$ indica que una parte de la población masculina se ha visto envuelta en procesos de mercantilización de su sexualidad (detrás del cual se dan diversos intercambios materiales y simbólicos que no solo incluyen dinero o bienes de consumo, sino también sentimientos de romance e intimidad).

Así pues, la historia de las prácticas sexuales que caracterizan a esta comunidad requiere ser entendida en función del turismo global, de las asimetrías económicas y de las diferencias raciales que de este surgen. En las narraciones de todos nuestros entrevistados, se hacía notar, ya fuera por el relato de 
experiencias personales o las de sus amigos, que el "meterse" con una turista puede ayudarle a uno a conseguir algo de dinero, un almuerzo o cervezas, o bien que "le pagaran la cuenta del celular [teléfono móvil] del mes". En este aspecto, los jóvenes encontraban un doble reto: primero, el de ser capaces de "acostarse" con mujeres de distintos lugares, de "ligarse" o de conquistar a una turista diferente cada día; y segundo, el de parecerles a ellas lo suficientemente atractivos para establecer relaciones íntimas "largas", o incluso "llegar a tener hijos". A lo interno de su grupo de pares, el superar exitosamente ambos retos supone acceder a estatus superior de masculinidad codiciado por los demás.

Los posibles efectos colaterales que podría acarrear este comportamiento (especialmente contagio de ITS) no aparecían dentro de sus narraciones como una preocupación aparente. En su lugar, se hablaba más bien en términos de una aventura o "un riesgo que vale la pena tomarse," algo que lo hacía a uno "sentirse vivo." En algunos casos, se incluía, además, dentro de sus estrategias de sobrevivencia, complementaria a sus actividades laborales, y de la cual sus propias familias también resultaban beneficiadas (en otras palabras, un medio de movilidad social que les permite a las personas pertenecer a un mundo de consumo que se les había sido negado anteriormente). Como vemos acá, la idea de riesgo adquiere un carácter positivo a contrapelo de la forma en que, tradicionalmente, se le ha pensado en el campo de la salud pública, es decir, como algo que genera ansiedades e incertidumbres.

Sobre este mismo punto, también se comentó que lo usual era hacerse de "novias extranjeras" por periodos que podían ir de quince días a un mes. Bajo estas circunstancias, lo ideal para los hombres era sostener relaciones monógamas temporales con las extranjeras, sin perder el vínculo con sus "novias" locales. El lapso de duración de sus relaciones con las extranjeras dependía del tipo de turista del cual habláramos (las que llegaban a la zona unos cuantos días, las que iban y venían, o bien, las que decidían quedarse a vivir allí).

En cuanto al tema del cuidado que había que tener al estar con una turista, se nos dijo que ellas los obligaban a utilizar condones (generalmente ellas los traían consigo), excepto en situaciones donde se presentaba consumo de alcohol y drogas, ya que allí, por lo general, todo el mundo se "descuidaba." Además, si con el paso del tiempo se desarrollaba un vínculo de confianza mayor con la extranjera, el uso obligatorio del condón dejaba de ser un requisito para tener sexo.

Las valoraciones hechas por nuestras entrevistadas sobre el turismo global variaron sustancialmente de las hechas por sus contrapartes masculinas. Si bien es cierto ellas centraron su atención en el vínculo establecido entre hombres locales y turistas, también fue posible recabar algunas menciones acerca de amigas suyas que se acostaban con "gente de afuera". Ellos, a 
cambio, se "portaban buena nota", es decir, se comportaban bien y les compraban "ropa, zapatos o algo", o bien, las invitaban a pasear a distintos lugares. En su opinión, este tipo de prácticas no era algo de lo cual habría que preocuparse. El sentir atracción sexual hacia un extranjero era normal, puesto que "en realidad los hombres locales no sirven para nada"; cuesta que puedan "sostener una relación" y, además, son parejas y padres irresponsables que no velan adecuadamente por ellas ni por los hijos que hayan tenido juntos:

He tenido, bueno, hace como una semana se acaba de ir un muchacho que es argentino y bueno, estuve hablando con él, tuve algo con él. En realidad los extranjeros así me llaman más la atención. Preferiría más un extranjero que una persona de aquí, pero uno nunca sabe (joven de Puerto Viejo, comunicación personal, 8 de marzo del 2013).

La posible mercantilización de relaciones de intimidad, en el caso de las mujeres, es un tema de estudio que requiere ser atendido a futuro. Por lo pronto y volviendo al tópico central de las conversaciones sostenidas con nuestras entrevistadas, las turistas suponían para ellas una competencia en lo que podríamos llamar el mercado sexual e íntimo de la zona. Acá, el trazado de fronteras simbólicas entre quienes podían confiar y quienes no era bastante claro. Las extranjeras no resultaban bienvenidas, en primera instancia, porque eran un grupo de personas con normas de comportamiento sexual distintas. Como nos fue comentado por una entrevistada:

[...] yo siento que las extranjeras son... como que viven la sexualidad más distinta que nosotros porque nosotros, no hacemos un montón de cosas que ellas si hacen, me entiendes [...] no sé, como que ellas son unas mujeres que tal vez les gusta más mamar, por ejemplo, que se la metan por detrás, nosotros tal vez somos como un poco como más recatadas en esas cosas [...] a lo que me han contado mis amigos, ellas son como las que se apuntan más a hacer esas cosas, entonces nosotros somos como que menos (joven de Puerto Viejo, comunicación personal, 8 de marzo del 2013).

Sin importar el tipo de prácticas sexuales que se saquen a colación, estas servían para juzgar lo "caliente" o "zorras" que resultaban ser las extranjeras. Asimismo, otra de sus características sería la de ser "liberadas", lo cual signi- 
ficaba que tenían una mayor independencia económica que las mujeres locales, así como una predisposición para ir a espacios públicos que tradicionalmente eran del dominio de los hombres. En este sentido, la búsqueda de encuentros sexuales se les facilitaba en tanto su capacidad de autosuficiencia, de no depender de nadie para moverse en la comunidad, así como la despreocupación que exhibían al asistir sin compañía a bares y discotecas.

De las turistas también debían cuidarse porque ejercían una influencia negativa directa en "sus hombres"; en especial, cuando se trataba de afrodescendientes. Las extranjeras alimentaban su ego machista al exaltar su físico, lo fuerte que eran, el tamaño de sus penes o el reafirmarlos como estupendos amantes, lo que en opinión de nuestras entrevistas reforzaba, a su vez, rasgos de personalidad nocivos (egoísmo, altanería, despreocupación hacia la pareja) que luego ellas tendrían que soportar al establecer relaciones íntimas con estos hombres. De nuevo, aparece aquí una aceptación implícita del tener que aguantar a su lado hombres que tenían parejas sexuales simultáneas. Si resultaba "normal" en el caso de las mujeres locales, también era de esperarse que sucediera con "las de afuera". El problema, pensando desde el punto de vista de factores de riesgo, era que nuestras entrevistadas rara vez se habían detenido a preguntarse si sus hombres utilizaban o no algún método profiláctico.

En este sentido, el riesgo se construía como algo externo a las relaciones sexuales que tenían con estos hombres. El problema, según nos fue explicado, no era ni el embarazo ni las ITS, sino que los hombres dejaban de estar con ellas al saber que podían "conseguirse" una extranjera. Eso les parecía más "excitante" e "interesante" por todo lo que ellas podían ofrecerles, no solo en la cama, sino fuera de ella. Si el lazo emocional con la turista se volvía muy fuerte, ellos llegaban a desestabilizarse anímicamente, dejando todo de lado, incluso sus responsabilidades paternas (en caso de tenerlas). Por ello, nuestras entrevistadas sentían que no eran capaces de "competir" ni en el campo económico ni en el campo amoroso con las turistas que visitaban la zona.

\section{Conclusiones}

Circunscribir la discusión que se desarrolla en el campo de la salud pública sobre riesgo y sexualidad a la noción de factores de riesgo, entendida única y exclusivamente en términos biomédicos, trae consigo ciertas ventajas y limitaciones. Por ejemplo: reduce la complejidad que tiene este fenómeno a una lista de conductas estándar que deben evitarse; crea instrumentos de medición generalizables a casi cualquier contexto y genera datos que permiten calcular y comparar en qué medida individuos y grupos sociales se alejan o acercan de ciertos parámetros ideales de seguridad. No obstante, analíticamente, la aproximación biomédica restringe la capacidad que tenemos de captar la manera en que las personas le han dado sentido a su vida sexual, 
lo cual dentro de esta concepción puede catalogarse como un comportamiento de riesgo, así como la valencia positiva o negativa que a ello se le pueda dar.

En vista de lo anterior, no abogamos, en el presente artículo, por hacer a un lado el análisis de este tipo comportamientos. Al contrario, se trata de reconocer, en primera instancia, que estos no constituyen meras opciones individuales, sino elecciones que la gente es llevada a asumir en virtud de normas y de valores compartidos. Al tratar las conductas asociadas a factores de riesgo como meras preferencias individuales, las biografías de las personas resultan abstraídas y removidas del mundo de prácticas y relaciones colectivamente orientadas que las han hecho posibles. Quienes estamos interesadas e interesados en coadyuvar al desarrollo de políticas en salud sexual para la persona joven tenemos por delante, justamente, la tarea de "devolverle su razón social de ser" a decisiones, tales como el estar con alguien que tiene múltiples parejas o el no darle importancia al uso de un condón en un momento dado.

En segundo lugar, resulta necesario hacer ejercicios analíticos que posibiliten dejar de pensar los riesgos como entidades objetivas, cuya amenaza una vez que sea conocida llevará a las personas por aquellos caminos racionales que les permitan sortearlos. Tal como puede deducirse de las entrevistas al grupo de jóvenes de Puerto Viejo, el debate público sobre riesgo y salud sexual se encuentra aún en ciernes. Por lo tanto, como parte de su desarrollo, es de esperar la confluencia de varios tipos de ideas y de creencias, desde aquellas que coinciden con la noción de factores de riesgo adoptada desde el sistema público de salud hasta formas de relacionarse con el cuerpo de sus parejas sexuales y el suyo propio, de crear lazos de intimidad, así como de generar dinámicas de rechazo y de atracción hacia determinadas personas, como es el caso de turistas que visitan esta comunidad.

Así pues, los conocimientos que pueden resultar irracionales, desde un punto de vista oficial o experto, no deberían ser catalogados como elementos distorsionadores de las acciones que, en buena teoría, deben seguir las personas, sino más bien como piezas clave que nos pueden permitir comprenderlas. De hecho, la interiorización de este tipo de saberes es algo complejo. En algunos momentos, tendrá sentido seguir sus recomendaciones, mientras que en otros no; puede suceder que este conocimiento sea tomado como algo irrelevante, a causa del evento que se vive. Esto podrá parecer un sinsentido; sin embargo, las personas tendrán sus razones. Asimismo, puede que el riesgo del cual habla un especialista no sea tan verdaderos para los demás. ¿Cuántas veces vemos que la gente tiene relaciones sin condón y aparentemente nada extraño les sucede? Entonces, podría perfectamente preguntarse sobre cuál sería la necesidad de alarmarse.

En vista de lo anterior, el tema de riesgo y de salud sexual requiere someterse a un debate público que les permita a diversos grupos poblacionales acce- 
der a diversas formas de conocimiento (incluyendo las aproximaciones biomédicas). En otras palabras, que dicho saber pueda cobrar sentido en su realidad inmediata y que le permita, a su vez, ir formando una perspectiva más reflexiva y crítica sobre el vínculo existente entre sus sexualidades y sus estados de salud. Lo peor que podría pasar en contextos como el del Caribe sur costarricense, espacio de encuentro multicultural que facilita el surgimiento de encuentros y relaciones afectivas y sexuales, sería seguir silenciando o estigmatizando el hecho de que la persona joven desarrolla una vida sexual que, más que racional, resulta razonable cuando se la analiza desde un punto de vista sociocultural.

\section{Referencias}

Adam, B. (2006). Infectious behaviour: imputing subjectivity to HIV transmission. Social Theory and Health, (4), 168-179.

Aggleton, P. y Parker, R. (2012). Introduction. En P. Aggleton y R. Parker (Eds.), Handbook of Sexuality, Health and Rights. New York: Routledge.

Alaszewski, A. y Brown, P. (2012). Making Health Policy. A Critical Introduction. Cambridge: Polity Press.

Almeida, N. \& Castiel, L.D. \& Ayres, J.R. (2009). Riesgo: concepto básico de la epidemiología. Salud Colectiva, 5(3), 323-344.

Beck, U. (1998). La Sociedad del Riesgo. Barcelona: Paidós.

Beck, U. (2009). World at risk. Cambridge: Polity.

Beck, U. (2011). Cosmopolitanism as imagined communities of global risk. American Behavioral Scientist, 55(10), 1346-1361.

Bloor, M. (1995). The Sociology of HIV Transmission. London: Sage.

Brown, P. y Heyman, B. (2012). Perspectives on 'the lens of risk' interview series: Interviews with Joost van Loon and Ortwin Renn. Health, Risk and Society, 14(5), 415-425.

Carmiol, E., Chacón, G., Vargas, y Walker, R. (2014). Caracterización del contexto comunitario, diagnóstico situacional, y componentes de línea base de la población meta. Limón: RET. 
CCSS. (2013a). Análisis de la Situación Integral de Salud 2011-2012 (actualizado 2013). Limón: Área de Salud de Talamanca.

CCSS. (2013b). Plan de Prevención en Salud 2013-2014. Limón: Área de Salud de Talamanca.

CCSS. (2014). Norma Nacional para la Atención Integral de la Salud de las Personas Adolescentes. San José: Dirección Garantía de Acceso a Servicios de Salud.

Douglas, M. (1994). Risk and Blame. Essays in Cultural Theory. London: Routledge.

Douglas, M. (1996). La aceptabilidad del riesgo según las ciencias sociales. Barcelona: Paidós.

Drew, K. (2012). The Cult and Science of Public Health. A Sociological Investigation. London: Blackwell.

Frohlick, S. (2012). Sexuality, Women, and Tourism. Cross-border desires through contemporary travel. London: Routledge.

Frohlick, S. (2013). Intimate Tourism Markets: Money, Gender, and the Complexity of Erotic Exchange in a Costa Rican Caribbean Town. Anthropological Quarterly, 86(1), 133-162.

Giddens, A. (2009). The Politics of Climate Change. Cambridge: Polity

Giddens, A. y Sutton, P. (2014). Conceptos esenciales de sociología. Madrid: Alianza Editorial.

INEC (2011). Censo Nacional de Población y Vivienda 2011. San José: Instituto Nacional de Estadística y Censos.

Ingham, R. y Aggleton, P. (2006). Promoting Young People's Sexual Health: International Perspectives. London: Routledge.

Jamieson, L. (2011). Intimacy as a concept: explaining social change in the context of globalization or another form of ethnocentricism? Social Research online,16(4), 1-15.

Lash, S. (2001). La reflexividad y sus dobles: estructura, estética, comunidad. En U. Beck, A. Giddens y S. Lash (Eds), Modernización reflexiva. Política, tradición y estética en el orden social moderno. Madrid: Alianza Universidad.

López-Ruiz, M. (2014). "Global Tourism, Local Economies of Desire, and Public Health: Barriers to Sexual Health Faced by Young Populations in 
a Poor Tourist Area in Costa Rica's Caribbean South" (ponencia presentada en el XXXII International Congress of the Latin American Studies Association (LASA), Chicago, IL, del 21 al 24 de mayo, 2014.

Lupton, D. (2012). Medicine as Culture. Illness, Disease and the Body. London: Sage.

Lupton, D. (2013). Risk. London: Routledge.

Martell, L. (2009). Global inequality, human rights and power: a critique of Ulrich Beck's cosmopolitanism. Critical Sociology, 35(2), 253-272.

Martínez, A. (2011). Antropología Médica. Teorías sobre la cultura, el poder y la enfermedad. Madrid: Alianza Editorial.

MINSA (2011). Informe de Resultados: Encuesta Nacional de Salud Sexual y Reproductiva, Costa Rica 2010. San José: Ministerio de Salud.

MINSA (2012). Líneas de Acción Estratégicas Área Rectora de Salud de Talamanca 2012. Limón: Área Rectora de Salud de Talamanca.

Molina, E. (2007). El encuentro entre lógicas globales y locales: empleo bananero y turístico en Cahuita y Puerto Viejo. Revista de Ciencias Sociales (117-118), 13-26.

OMS (2009). Global health Risks. Mortality and Burden of Disease Attributable to Selected Major Risks. Genova: World Health Organization.

Porta, M. (2013). A Dictionary of Epidemiology. Cambridge: Oxford University Press.

Porter, D. (1999). Health, Civilization and the State. A history of public health from ancient to modern times. London: Routledge.

Rasborg, K. (2012). World risk society or new rationalities of risk? A critical discussion of Ulrich Beck's theory of reflexive modernity. Thesis Eleven, 108(1), 3-25.

Schneider, M.J. (2011). Introduction to Public Health. Boston: Jones and Bartlett Publishers.

Smallman, S. (2007). The AIDS Pandemic in Latin America. Chapel Hill: The University of North Carolina Press.

UNFPA. (2013). Estrategia Regional de UNFPA sobre Adolescencia y Juventud para América Latina y el Caribe. Santiago: UNFPA. 
UNFPA (2014). The State of World Population 2014, The Power of 1.8 Billion: Adolescents, Youth and the Transformation of the Future. Santiago: UNFPA.

Zelizer, V. (2007). The Purchase of Intimacy. New Jersey: Princeton University Press. 